\title{
Energy-Efficient Two-Hop LTE Resource Allocation in High Speed Trains with Moving Relays
}

\author{
Ahmad Alsharoa*, Hakim Ghazzai*, Elias Yaacoub ${ }^{\dagger}$, and Mohamed-Slim Alouini*, \\ ${ }^{*}$ Computer, Electrical, and Mathematical Science of Engineering (CEMSE) Division, \\ King Abdullah University of Science and Technology (KAUST), \\ Thuwal, Makkah Province, Kingdom of Saudi Arabia, \\ E-mails: \{ahmad.sharoa, hakim.ghazzai, slim.alouini\}@kaust.edu.sa. \\ ${ }^{\dagger}$ Qatar Mobility Innovations Center (QMIC), Qatar Science and Technology Park, Doha, Qatar, \\ E-mail: eliasy@qmic.com.
}

\begin{abstract}
High-speed railway system equipped with moving relay stations placed on the middle of the ceiling of each train wagon is investigated. The users inside the train are served in two hops via the 3GPP Long Term Evolution (LTE) technology. The objective of this work is to maximize the number of served users by respecting a specific quality-of-service constraint while minimizing the total power consumption of the eNodeB and the moving relays. We propose an efficient algorithm based on the Hungarian method to find the optimal resource allocation over the LTE resource blocks in order to serve the maximum number of users with the minimum power consumption. Moreover, we derive a closed-form expression for the power allocation problem. Our simulation results illustrate the performance of the proposed scheme and compare it with various previously developed algorithms as well as with the direct transmission scenario.
\end{abstract}

Index Terms-High-speed railway communication, moving relays, LTE networks, resource allocation algorithm.

\section{INTRODUCTION}

High-speed public transportation such as train, tram, and bus passengers may want to invest their riding time by catching up their works or enjoying broadband Internet. The 3rd Generation Partnership Project (3GPP) has supported relay communications in the Long Term Evolution (LTE) release-10 standard [1].

The idea of using Moving Relays (MRs) within the highspeed railway mobile communication has been proposed recently to enhance the cellular coverage and overcome the moderate to high Vehicle Penetration Loss (VPL) [2]. In [2], it has been shown that when VPL is moderate to high, the performance of MRs transmission outperforms the performance of fixed relays transmission as well as direct transmission (i.e., without a relay assistance). VPL can be negligible by introducing two antennas connected together by a wired cable; outside and inside the vehicle. The outside antenna communicates with the LTE eNodeB (eNB), while the inside antenna communicates with the User Equipments (UEs) inside the vehicle [3], [4]. The capacity and handover performance

The work of A. Alsharoa, H. Ghazzai, and M.-S. Alouini was funded by a grant from King Abdulaziz City of Science and Technology (KACST). The work of E. Yaacoub was funded by NPRP grant \#4-347 -2 - 127 from the Qatar National Research Fund (a member of Qatar Foundation). The statements made herein are solely the responsibility of the authors. gain for high-speed railway with and without relay assistance have been investigated in [3]. High-speed LTE MRs resource allocation with fixed power assigned to each resource block has been investigated in [5]. In the latter work, the authors proposed a simple iterative resource allocation approach in order to maximize the total sum rate of the UEs. However, the aforementioned works have not discussed the resource allocation problem for high-speed railway communication.

In this paper, we investigate the resource allocation problem for high-speed railway communication assisted by LTE MRs. We start by formulating an optimization problem that maximizes the number of served UEs while minimizing the total power consumption of the system, taking into account the power budget and respecting a certain Quality-of-Service (QoS) for each UE. More specifically, the data rate of each UE is restricted to be greater than a pre-defined data rate threshold. Then, we derive a closed-form expression for the power allocation solution assuming fixed Resource Blocks (RBs) allocation. Afterwards, we propose a practical resource allocation approach based on Hungarian algorithm to find the optimal power allocation at each RB assigned to each UE. Finally, we compare the performance of our proposed scheme with some algorithms developed in the literature and show that our approach maintains the system QoS even for longrange distance.

The rest of this paper is structured as follows. Section II presents the system model. The optimization problem is formulated in Section III. Section IV describes the proposed algorithm. Simulation results are discussed in Section V. Finally, the paper is concluded in Section VI.

\section{SyStem MOdEL}

We consider a high-speed train consisting of $L$ wagons with $M$ UEs inside the train distributed randomly over the train wagons. We assume that during an interval of time, the train is moving within a LTE cell. The UEs are communicating with the LTE eNB that occupies the center of the cell via MR stations located at the top of each wagon. Each MR station is composed of two antennas connected together by a cable and placed outside and inside the train wagon as shown in Fig.1.

In LTE, the available spectrum is divided into RBs consisting of 12 adjacent subcarriers. Each RB has a bandwidth 
of $B_{R B}=180 \mathrm{KHz}$ while each subcarrier has a bandwidth of $B_{\text {sub }}=15 \mathrm{KHz}$ [6]. In our framework, we consider an orthogonal LTE transmission where the total bandwidth of $B_{T}=20 \mathrm{MHz}$ is subdivided into three blocks. The first block of $10 \mathrm{MHz}$ (equivalent to 50 orthogonal RBs) is owned by the eNB, while the other blocks, each of $5 \mathrm{MHz}$ (equivalent to 25 orthogonal RBs), are owned by two MRs $i$ and $i+1, \forall i=1, \cdots, L-1$ [6], [7]. Hence, the same frequency blocks are reused with two consecutive MRs. In this case, we can assume that all UEs in wagon $i$ are protected from the co-channel interference caused by MRs in wagons $i-2$ and $i+2$ due to the wagon penetration loss and to the distance separating two wagons using the same frequency.

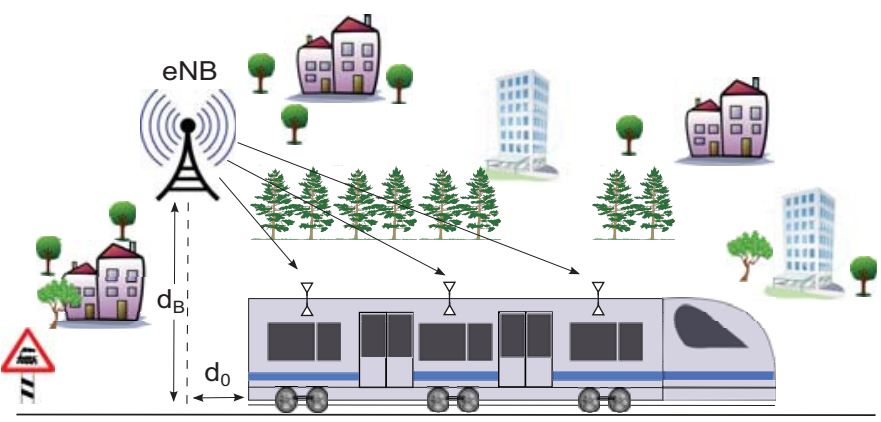

Fig. 1 High-Speed Train System Model.

In the first hop, also known as the backhaul link, the eNB transmits different signals over different RBs to the outside antennas of the MRs. Consequently, in the second hop, also know as the access link, the $i^{\text {th }}$ MR serves the $M_{i}$ UEs inside the $i^{t h}$ train car such that $\sum_{i=1}^{L} M_{i}=M$. The transmitter $t$ power vector is denoted $\boldsymbol{P}_{t}=\left[P_{t}^{(1)}, \ldots, P_{t}^{(k)}, \ldots, P_{t}^{(K)}\right]$, where $K$ is the number of RBs and equal to 50 for the backhaul link and 25 for the access link. In our study, we propose to compare this high-speed train communication model using MRs with the direct high-speed train communication scenario where the users inside the train are communicating directly with the eNB via the total bandwidth $B_{T}=20 \mathrm{MHz}$ (equivalent to 100 orthogonal RBs).

\section{A. Path Loss Analysis for Relay Transmission (eNodeB-Relay and Relay-User)}

The Path Loss (PL) used in the backhaul link between the eNB and the $i^{t h}$ MR (eNB-MR link) over the $k^{t h} \mathrm{RB}$ under D1 Line of Sight (LoS) propagation scenario [8] is denoted as $P L_{B-R_{i}}^{(k)}$ and given in (1), where $d_{B-R_{i}}$ is the distance from the eNB to the $i^{t h}$ MR in meter $\mathrm{m}, f^{(k)}$ is the center frequency over the $k^{t h} \mathrm{RB}$ in $\mathrm{Hz}, h_{B}$ and $h_{T}$ are the height of the eNB and the train in $\mathrm{m}$, respectively, and $d_{B P}=4 h_{B} h_{T} f^{(k)} / c$ is the breaking point distance, where $c=3 \times 10^{8} \mathrm{~m} / \mathrm{s}$ is the speed of light in vacuum.

The PL used in the access link between the $i^{t h} \mathrm{MR}$ and the $m^{t h}$ UE (MR-UE link) belonging to the $i^{t h}$ wagon over the $k^{t h} \mathrm{RB}$ under A1 indoor LoS propagation scenario [8] is given by

$$
\begin{aligned}
P L_{R_{i}-U_{m}}^{(k)}\left(d_{R_{i}-U_{m}}\right)= & 46.4+18.7 \log _{10}\left(d_{R_{i}-U_{m}}\right)+ \\
& 20 \log _{10}\left(f^{(k)}[\mathrm{GHz}] / 5\right),
\end{aligned}
$$

where $d_{R_{i}-U_{m}}$ is the distance from the $i^{t h}$ MR to the belonging $m^{t h} \mathrm{UE}$ in $\mathrm{m}$.

\section{B. Path Loss Analysis for Direct Transmission (eNodeB-User)}

In this section, the PL of the direct link between eNB and UE without relay assistance under outdoor-indoor propagation scenario [8] is given by

$$
\begin{aligned}
P L_{B-U_{m}}^{(k)}\left(d_{U_{m}, \text { out }}, d_{U_{m}, \text { in }}\right)= & P L_{B-R}^{(k)}\left(d_{U_{m}, \text { out }}\right)+ \\
& P L_{R-U}^{(k)}\left(d_{U_{m}, \text { in }}\right)+P L_{\text {outer }},
\end{aligned}
$$

where $d_{U_{m} \text {,out }}$ and $d_{U_{m}}$, in are the distance between the eNB and the closest point of the train wall to the $m^{t h} \mathrm{UE}$ and the distance from train wall to the $m^{t h}$ UE in $\mathrm{m}$, respectively. $P L_{\text {outer }}=W_{e}+W G_{e}(1-\cos (\theta))^{2}$ is the path loss through the outer wall, where $W_{e}$ is the loss through the train wall for the perpendicular penetration, while $W G_{e}$ is the loss through the train wall for the parallel penetration, and $\theta$ is the angle between the normal of the train wall and the outgoing (incoming) ray.

It is assumed that all the aforementioned formulas are generalized for the frequency range $2-6 \mathrm{GHz}$ (for more details refer to [8]).

\section{Channel Model}

The channel model in $[\mathrm{dB}]$ over the $k^{t h} \mathrm{RB}$ is a function of the PL between transmitter $t$ and receiver $r$, shadowing, and fading as follows

$$
H_{t, r, \mathrm{~dB}}^{(k)}=-P L_{t, r}^{(k)}-\xi_{t, r}+20 \log _{10}\left(F_{t, r}^{(k)}\right),
$$

where $\xi_{t, r}$ is the log-normal shadowing between $t$ and $r$ with a standard deviation equal to $\sigma$ and $F_{t, r}^{(k)}$ corresponds to the fast Rayleigh fading power between $t$ and $r$ such that $\mathbb{E}\left[|a|^{2}\right]=1$, where $a$ is a Rayleigh parameter and $\mathbb{E}[$.$] is the expectation$ operator. In this case of fast Rayleigh fading, we consider that the channels between eNB and UEs are constant during the channel de-correlated time $T_{d}$.

In (4), under the spatial log-normal shadowing assumption, the shadowing correlation is taken into account where the spatial correlation can be, indeed, used to measure how fast the local mean power evolves as the vehicle moves along a certain route [9]. Therefore, the shadowing correlation model for moving transmitter $t$ and receiver $r$ can be given by an exponential function [9], [10] as follows

$$
\Lambda_{\xi}\left(\Delta d_{t}, \Delta d_{r}\right)=\exp \left(-\frac{\Delta d_{t}+\Delta d_{r}}{l_{c}}\left(\log _{e} 2\right)\right),
$$

where $\exp ($.$) denotes the exponential function, \Delta d_{t}$ and $\Delta d_{r}$ represent the movements of the transmitter and receiver, respectively, and $l_{c}$ is the decorrelation length which depends on the environment (equal to 20 meters in the vehicular test environment [11]). Consequently, we can use (5) to determine the shadowing values as follows

$$
\begin{aligned}
\xi_{t, r}\left(d_{t}+\Delta d_{t}, d_{r}+\Delta d_{r}\right)= & \xi\left(d_{t}, d_{r}\right) \Lambda_{\xi}\left(\Delta d_{t}, \Delta d_{r}\right)+ \\
& \left(1-\xi\left(\Delta d_{t}, \Delta d_{r}\right)\right) \xi_{t, r}^{\text {new }}
\end{aligned}
$$




$$
P L_{B-R_{i}}^{(k)}\left(d_{B-R_{i}}\right)= \begin{cases}44.2+21.5 \log _{10}\left(d_{B-R_{i}}\right)+20 \log _{10}\left(f^{(k)}[\mathrm{GHz}] / 5\right), & 10 \mathrm{~m}<d_{B-R_{i}}<d_{B P}, \\ 10.5+40.0 \log _{10}\left(d_{B-R_{i}}\right)-18.5 \log _{10}\left(h_{B}\right)- & \\ 18.5 \log _{10}\left(h_{T}\right)+1.5 \log _{10}\left(f^{(k)}[\mathrm{GHz}] / 5\right), & d_{B P}<d_{B-R_{i}}<10 \mathrm{~km},\end{cases}
$$

where the shadowing value at positions $\left(d_{t}+\Delta d_{t}, d_{r}+\right.$ $\Delta d_{r}$ ) has a component equal to the shadowing value at positions $\left(d_{t}, d_{r}\right)$, with the amount of similarity determined by $\Lambda_{\xi}\left(\Delta d_{t}, \Delta d_{r}\right)$, in addition to an independent shadowing component corresponding to the new location $\xi_{t, r}^{\text {new }}$. In our system model, we assume that $\Delta d_{t}=0$ since the eNB has a fixed position.

\section{PROBlem Formulation}

In this section, our objective is to find the optimal resource allocation in terms of RBs and power at the eNB and MRs in order to maximize the number of served UEs with the minimum power consumption. A receiver $r$ (i.e., a MR or UE depending on the link) is considered successfully served if its data rate $R_{r} \geq R_{t h}^{r}$ where $R_{r}$ is given by

$$
R_{r}\left(\boldsymbol{P}_{t}\right)=\sum_{k \in \mathcal{I}_{R B, r}} B_{R B} \log _{2}\left(1+\gamma_{t, r}^{(k)}\left(P_{t}^{(k)}\right)\right),
$$

where $\mathcal{I}_{R B, r}$ is the set of RBs allocated to receiver $r . R_{t h}^{r}$ corresponds to the data rate threshold of the receiver $r$. In (7), $\gamma_{t, r}^{(k)}$ is the received signal-to-interference-plus-noise ratio at the receiver over the $k^{\text {th }} \mathrm{RB}$ and is given as follows

$$
\gamma_{t, r}^{(k)}\left(P_{t}^{(k)}\right)=\frac{P_{t}^{(k)} H_{t, r}^{(k)} G_{t} G_{r}}{I+N_{0} B_{R B}},
$$

where $G_{t}$ and $G_{r}$ are the transmitter and receiver antenna gains, respectively. $I$ and $N_{0}$ are the average co-channel interference caused by other eNBs and/or other UEs and the noise variance, respectively. Thus, our optimization problem for each backhaul or access link can be formulated as

$$
\underset{\boldsymbol{\epsilon}, \boldsymbol{P}_{t} \geq 0}{\operatorname{minimize}} \sum_{k=1}^{K} P_{t}^{(k)} \sum_{r=1}^{M_{i}, L} \epsilon(k, r)
$$

subject to

(C1: Power budget constraint at the transmitter $t$ ):

$$
\sum_{k=1}^{K} P_{t}^{(k)} \leq \bar{P}_{t}
$$

(C2: Receiver $r$ rate constraint):

$$
R_{r}\left(\boldsymbol{P}_{t}\right) \geq R_{t h}^{r}, \quad \forall r,
$$

(C3: RB selection constraints):

$$
\sum_{r=1}^{M_{i}, L} \epsilon(k, r) \leq 1, \quad \forall k, \text { and } \sum_{k=1}^{K} \epsilon(k, r) \leq\left|\mathcal{I}_{R B, r}\right|, \quad \forall r,
$$

where $\bar{P}_{t}$ is the peak power at the transmitter $t . \epsilon$ is either a matrix of size $K \times L$ such that $K \geq L$ if we consider the backhaul link or $K \times M_{i}$ such that $K \geq M_{i}$ if we consider the access link inside wagon $i$. Binary variables $\epsilon(k, r)$ refer to the status of the $k^{\text {th }} \mathrm{RB}$ whether it is allocated to receiver $r$ or not and it is given as follows

$$
\epsilon(k, r)= \begin{cases}1, & \text { if } k^{t h} \mathrm{RB} \text { is allocated to receiver } r, \\ 0, & \text { otherwise. }\end{cases}
$$

Constraint (11) ensures that the QoS of the receiver $r$ is satisfied, while constraint (12) indicates that each RB can be allocated to only one UE and, at maximum, one UE can be served by $\left|\mathcal{I}_{R B, r}\right|$ RBs where $|\mathcal{X}|$ denotes the cardinality of the set $\mathcal{X}$. For instance, in the access link, we assume $\left|\mathcal{I}_{R B, r}\right|=1$ (i.e., a user is served only by a unique RB) while in the backhaul link, as the rate is expected to be high, we can allocate more than one RB to each MR. Note that, in LTE, the allocated RBs to a receiver have to be adjacent [6], [7]. The optimal solution for our formulated optimization problem given in (9) is difficult to find and considered as NP-hard problem due to the existence of the binary variables $\epsilon(k, r)$ [12]. However, if we pre-define the RB allocation over the receivers (i.e., $\epsilon$ is known), we can solve our problem efficiently by using the Lagrangian method which consists in computing the corresponding Lagrangian function $\mathcal{L}$ [13]. Therefore, we can solve our optimization problem by exploiting its strong duality represented by the following optimization problem [13]:

$$
\max _{\boldsymbol{\lambda} \geq 0} \min _{\boldsymbol{P}_{t} \geq 0} \mathcal{L}\left(\boldsymbol{\lambda}, \boldsymbol{P}_{t}\right) .
$$

where $\lambda$ is the vector that contains all the Lagrangian multipliers of the problem. The Lagrangian expression is derived as follows

$$
\begin{aligned}
\mathcal{L}\left(\boldsymbol{\lambda}, \boldsymbol{P}_{t}\right) & =\sum_{k=1}^{K} P_{t}^{(k)}+\lambda_{0}\left[\sum_{k=1}^{K} P_{t}^{(k)}-\bar{P}_{t}\right] \\
& +\sum_{r=1}^{M_{i}, L} \lambda_{r}\left[R_{t h}^{r}-R_{r}\left(\boldsymbol{P}_{t}\right)\right] .
\end{aligned}
$$

where $\lambda_{0}$ and $\lambda_{r}$ represent the Lagrangian multipliers related to the peak power constraint and the lower rate bound constraint at the receiver $r$, respectively. By taking the derivative of the Lagrangian with respect to the $P_{t}^{(k)}$ where $k=1, \ldots, K$, we can find the optimal transmit power allocated over the $k^{t h} \mathrm{RB}$ that minimizes the Lagrangian function as well as the power consumption of the transmitter. The optimal power expression over the $k^{t h} \mathrm{RB}$ is given as

$$
P_{t}^{(k)}=\left(\frac{\lambda_{r_{k}} B_{R B}}{\left(2 \log _{e} 2\right)\left(1+\lambda_{0}\right)}-\frac{I+N_{0} B_{R B}}{H_{t, r}^{(k)} G_{t} G_{r}}\right)^{+}
$$

where $(x)^{+}$denotes the maximum between $x$ and zero and $\lambda_{r_{k}}$ is the Lagrangian multiplier associated to receiver $r$ served by RB $k$ since from constraint (12), each RB serves only one receiver. In order to solve this analytical expression based on $\lambda$, we can employ the subgradient method or other heuristic approaches to find the optimal Lagrangian multipliers of this problem [14]. 
The challenge now is to enhance the performance of the system by optimizing the powers simultaneously with the RBs allocation.

\section{Proposed Algorithm}

In order to solve the optimization problem formulated in (9)-(12), we proceed as follows: First, we focus on the access link to find the optimal resource allocation at each MR station. Depending on the number of UEs $M_{i}$ per wagon $i$ and the corresponding target data rate $R_{t h}^{U}$, we can compute the target data rate per each MR station denoted $R_{t h}^{R_{i}}, \forall i=1, \cdots, L$, which is equivalent to the sum of the target rates of the UEs it is serving. Once $R_{t h}^{R_{i}}$ becomes known for each MR $i$, we can then focus on the backhaul link and find the optimal resource allocation at the eNB.

In this access link, we assume that $\left|\mathcal{I}_{R B, r}\right|=1$. Indeed, in practical scenario, for this short range link, one RB per UE is enough to achieve the target data rate. The steps of the proposed approach for the resource allocation algorithm can be described as follows:

- Step 1: Simplify the optimization problem by distributing the peak power of the $i^{\text {th }}$ MR uniformly over its belonging RBs (i.e., $\bar{P}_{R_{i}}^{(k)}=\frac{\bar{P}_{R_{i}}}{K}$ ) where $\bar{P}_{R_{i}}^{(k)}$ is the peak transmit power at the $k^{t h} \mathrm{RB}$ of the $i^{\text {th }}$ MR. This means that constraint (10) becomes as follows

$$
P_{R_{i}}^{(k)} \leq \bar{P}_{R_{i}}^{(k)}, \forall k=1, \ldots, K .
$$

Thus, the solution of the optimization problem becomes as follows:

$$
P_{R_{i}}^{(k)}= \begin{cases}\frac{A_{t h}}{H_{R_{i}, U_{m}}^{(k)}}, & \text { if } H_{R_{i}, U_{m}}^{(k)} \geq \frac{A_{t h}}{\bar{P}_{R_{i}}^{(k)}} \\ 0, & \text { otherwise }\end{cases}
$$

where $A_{t h}=\left(2^{\frac{R_{t h}^{U}}{B_{R B}}}-1\right)\left(I+N_{0} B_{R B}\right)$. The obtained solution derived in (18) means that the UE $m$ served by the $i^{t h}$ MR over subcarrier $k$ can achieve its data rate only if the corresponding channel is relatively good.

- Step 2: Compute $P_{R_{i}}^{(k)}$ for all possible (RB, UE) combinations (i.e., $K \times M_{i}$ possibilities) inside the $i^{t h}$ wagon.

- Step 3: Employ a combinatorial optimization approach entitled the Hungarian algorithm [15] to find the best (RB, UE) combinations that maximizes the total number of served UEs with minimum power consumption inside the $i^{\text {th }}$ wagon. However, in some cases due to the modification of constraint (12), this method with the Hungarian algorithm is not enough to serve the maximum number of UEs. Indeed, after allocating the RBs, some UEs may not achieve the required rate because of the power limitation as expressed in (17).

- Step 4: If for the wagon $i$, the number of served UEs is less than $M_{i}$, redistribute uniformly the remaining power over the remaining RBs $\left(\bar{P}_{R_{i}}=\bar{P}_{R_{i}}-\sum_{k=1}^{K} P_{R_{i}}^{(k)}\right)$ and repeat Steps 1 to 3 for the non-served UEs in wagon $i$. In fact, the peak power per RB may increase comparing to Step 1.

- Step 5: Repeat Step 4 until serving all UEs or the remaining power per $\mathrm{RB}$ is not enough to achieve the
UE target data rate. In the latter case, try to serve at least one UE among of the remaining UEs by allocating the total remaining power to the UE having the best channel gain.

Note that the proposed approach is applied for the $L$ wagons simultaneously. At the end of the algorithm, $R_{t h}^{R_{i}}, \forall i=$ $1, \cdots, L$, becomes known and the eNB applies the same approach but for the backhaul link. However, if at least one of the MRs is not served, we eliminate some UEs in the corresponding wagon in order to decrease the target data rate of the MR and thus try to serve the maximum number of UEs in that wagon. Details of the proposed algorithm are summarized in Algorithm 1 by replacing $Q$ and $t$ either by $M_{i}$ and $R_{i}$ for the access link or by $L$ and $B$ for the backhaul link, respectively.

Algorithm 1 Resource Allocation Algorithm for High-Speed Train Communication with Moving Relays

1: Initialize $\mathcal{Q}=\{1, \ldots, Q\}, \mathcal{K}=\{1, \ldots, K\}, Q_{\text {served }}=0$.

2: repeat

3: Compute the transmit powers $P_{t}^{(k)}$ as it is given in (18) for each $(k, r) \in(\mathcal{K}, \mathcal{Q})$ pairs.

4: Find $\left(k^{*}, r^{*}\right)$ combinations by employing the Hungarian algorithm in order to serve the maximum number of UEs with minimum power consumption.

5: $\quad$ Mark $\left(k^{*}, r^{*}\right)$ combinations as occupied (i.e., update $\boldsymbol{\epsilon}$ ).

6: $\quad \mathcal{Q}=\mathcal{Q} \backslash\left\{r^{*}\right.$ 's $\}, \mathcal{K}=\mathcal{K} \backslash\left\{k^{*}\right.$ 's $\}$ and $Q_{\text {served }}=Q-|\mathcal{Q}|$.

7: $\quad \bar{P}_{t}=\bar{P}_{t}-\sum_{k \in \mathcal{K}} P^{(k)}$.

8: until $\left(Q_{\text {served }}=Q \| \bar{P}_{t}\right.$ remains constant $)$.

9: if $\left(Q_{\text {served }}<Q\right)$ then

10: $\quad$ while $\exists(k, r) \in \mathcal{Q} \times \mathcal{K}$ such that $H_{t, r}^{(k)} \geq \frac{A_{t h}}{P_{t}}$ do

11: $\quad H_{t, r_{b}}^{\left(k_{b}\right)}=\max _{\left(r_{b}, k_{b}\right) \in(\mathcal{Q} \times \mathcal{K})} H_{t, r}^{(k)}$.

12: Find the transmit power $P_{t}^{\left(k_{b}\right)}$ corresponding to $r_{b}$ by computing (18).

13: $\quad$ Mark $\left(k_{b}, r_{b}\right)$ pair as occupied (i.e., update $\left.\boldsymbol{\epsilon}\right)$.

14: $\mathcal{Q}=\mathcal{Q} \backslash\left\{r_{b}\right\}, \mathcal{K}=\mathcal{K} \backslash\left\{k_{b}\right\}$ and $Q_{\text {served }}=Q-|\mathcal{Q}|$.

15: $\quad \bar{P}_{t}=\bar{P}_{t}-\sum_{k \in \mathcal{K}}^{|\mathcal{K}|} P^{(k)}$.

16: end while

17: end if

Once we find the allocation matrix $\epsilon^{*}$ and the corresponding total power consumption using our proposed approach, we compare the obtained results with the optimal analytical solution given in (16) for the same allocated $\epsilon^{*}$. As will be shown in the sequel, the proposed approach achieves very close performance comparing with the optimal solution for the same $\epsilon^{*}$.

\section{SIMULATION RESULTS}

In this section, we consider a high-speed train scenario where the train is constituted of $L=10$ wagons of $10 \mathrm{~m}$ length, $5 \mathrm{~m}$ width, and $2.5 \mathrm{~m}$ height. Each UE communicates with the eNB via one MR placed on the middle of the ceiling of each wagon. We assume that accurate channel state information can be available at the transmitters, even at high-speeds, using for example the predictor antenna method described in [16]. The resource allocation algorithm proposed in Section 
IV is applied in both access and bakchaul links using the parameters summarized in Table I. Also, it is assumed that the de-correlated time $T_{d}=1 \mathrm{~ms}[17]$ and the target data rate $\left(R_{t h}^{U}\right)$ is the same for all UEs. Initially, the distance separating the eNB and the railway is equal $d_{B}=100 \mathrm{~m}$ while the end of the train is located at the distance $d_{0}=0 \mathrm{~m}$ as shown in Fig.1. Furthermore, we apply the proposed algorithm to the direct transmission where each UE communicates directly with the eNB without assistance from the relay.

TABLE I: SIMULATION PARAMETERS

\begin{tabular}{|l|l|}
\hline Parameter & Value \\
\hline \hline Train speed & $350 \mathrm{~km} / \mathrm{hr}$ \\
\hline Carrier frequency & $2.6 \mathrm{GHz}$ \\
\hline Total System Bandwidth $\left(B_{T}\right)$ & $20 \mathrm{MHz}$ \\
\hline eNB and MR $i$ transmit antenna gains $\left(G_{B}, G_{R_{i}}\right)$ & $(14,0) \mathrm{dBi}$ \\
\hline MR $i$ and UE $m$ receive antenna gains $\left(G_{R_{i}}, G_{U_{m}}\right)$ & $(0,0) \mathrm{dBi}$ \\
\hline Total number of UEs $(M)$ & 250 \\
\hline Total number of UEs per wagon $\left(M_{i}\right)$ & 25 \\
\hline Peak power at each $i^{t h}$ MR $\left(\bar{P}_{R_{i}}\right)$ & $20 \mathrm{dBm}$ \\
\hline Total transmit peak power $\left(\bar{P}=\bar{P}_{B}-\sum_{i=1}^{L} \bar{P}_{R_{i}}\right)$ & $46 \mathrm{dBm}$ \\
\hline Shadowing standard deviation $(\sigma)$ & $8 \mathrm{~dB}$ \\
\hline Perpendicular penetration train loss $\left(W_{e}\right)$ & $18 \mathrm{~dB}$ \\
\hline Parallel penetration train loss $\left(W G_{e}\right)$ & $15 \mathrm{~dB}$ \\
\hline Average co-channel interference $(I)$ & $-110 \mathrm{dBm}$ \\
\hline Average noise power variance at room temperature $\left(N_{0}\right)$ & $-174 \mathrm{dBm}$ \\
\hline
\end{tabular}

In all the following simulation results, we compare our proposed algorithm to the Hungarian method which corresponds to Steps 1 to 3 only and to an iterative algorithm proposed in [5]. The latter method consists in allocating RBs to destinations in a way to maximize the backhaul performance by selecting the best RB for each destination iteratively (i.e., best channel gain).

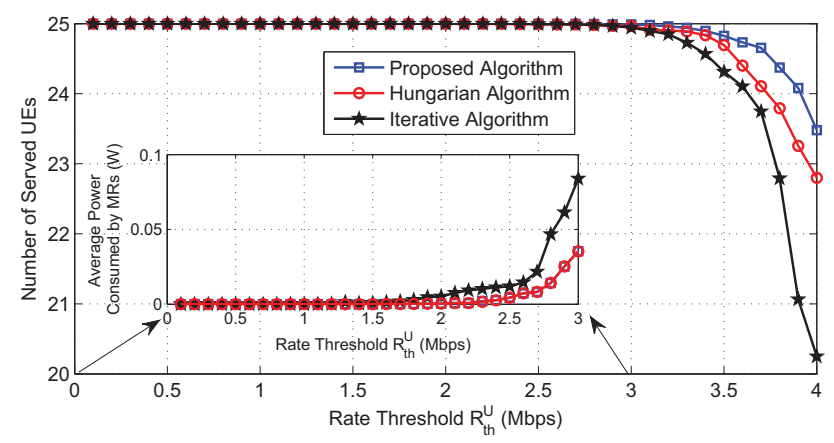

Fig. 2 Average number of served UEs versus the $R_{t h}^{U}$ Mbps in the access link.

Fig.2 plots the average number of served UEs versus the target data rate $R_{t h}^{U}$ for the access link (MR-UE link). It can be shown that, for all algorithms, the MRs can successfully serve the 25 UEs up to a certain $R_{t h}^{U}=3 \mathrm{Mbps}$. It is noticed that the proposed and Hungarian approaches outperform the iterative algorithm in terms of power consumption.

In the sequel, we investigate the performance of the proposed algorithm for full system operation (i.e., backhaul and access links). Fig.3 illustrates the number of served UEs as a function of the distance $d_{0}$ for a fixed UE target data rate $R_{t h}^{U}=0.5$ Mbps. Note that, when all users in a wagon $i$ are successfully served, the $i^{\text {th }}$ MR target data rate becomes

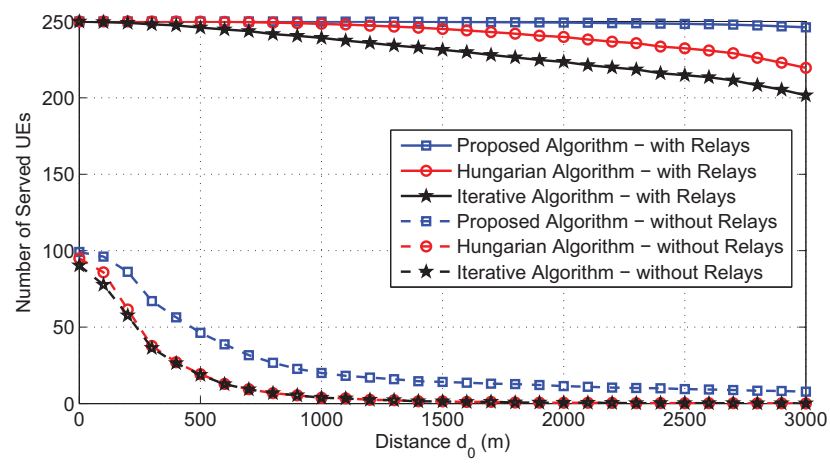

Fig. 3 Total number of served UEs versus the distance $d_{0}$ for $R_{t h}^{U}=0.5$ Mbps.

equal to 12.5 Mbps. Fig. 3 shows that the performance of our proposed algorithm outperforms the other algorithms for both relay and direct transmissions. As the train moves away from the eNB, the advantages of our proposed algorithm appear clearly: In the relay transmission, the algorithm maintains the system QoS even for long-range distance, e.g., for $d_{0}=2000$ $\mathrm{m}$ where our proposed approach still serves all the 250 UEs while the Hungarian and iterative algorithms can only serve around 240 and 223 UEs, respectively. Also, we notice the importance of the introduction of relays at the top of the train. Relays can not only help in coping with the pathloss increase but also in serving more UEs as they offer more available RBs. Indeed, with direct transmission and with $B_{T}=20 \mathrm{MHz}$, the proposed algorithms can at maximum serve 100 UEs while thanks to the relays, the eNB is able to communicate with 250 UEs.
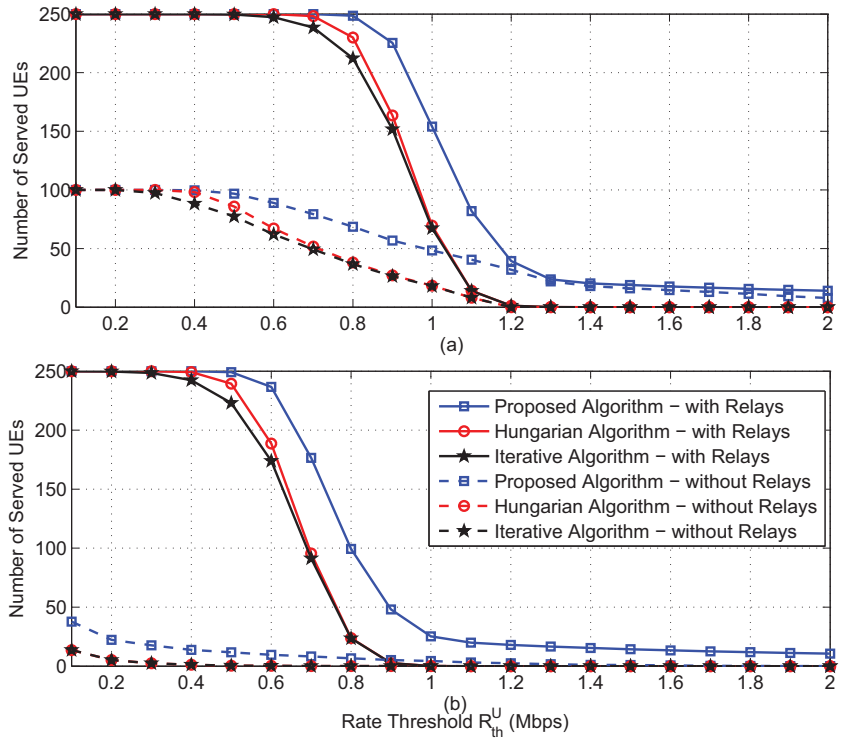

Fig. 4 Total number of served UEs versus $R_{t h}^{U}$ for (a) distance $d_{0}=100 \mathrm{~m}$, (b) distance $d_{0}=2000 \mathrm{~m}$.

Fig.4 depicts the impact of the $R_{t h}^{U}$ on the number of served UEs for fixed values $d_{0}=\{100,2000\} \mathrm{m}$. It is noticed that all algorithms can serve all UEs up to a certain $R_{t h}^{U *}$. For instance, for $d_{0}=100 \mathrm{~m}, R_{t h}^{U^{*}}=\{0.8,0.7,0.5\} \mathrm{Mbps}$ using the proposed approach, Hungarian method and iterative 
algorithm, respectively. This can be justified by the fact that the power budget is only enough for low $R_{t h}^{U}$. As the required $R_{t h}^{U}$ increases, the benefit of using our proposed algorithm is noticed. For instance, for $R_{t h}^{U}=0.6 \mathrm{Mbps}$ and $d_{0}=2000$ $\mathrm{m}$, the proposed algorithm can serve around 240 UEs while the other algorithms can only serve 190 and 175 UEs as shown in Fig.4(b). On the other hand, it is clear that the relay transmission (solid lines) outperforms the direct transmission (dashed lines). However, we notice a significant decreasing slope of the number of served UEs with the relay transmission comparing to the direct transmission. Indeed, if one relay is not served this means that the 25 UEs in the corresponding wagon are not served even after employing Step 5 of the proposed approach while with the direct transmission the increase of the target data rate per UE eliminates UE by UE. The increase of the distance $d_{0}$ implies a decrease of the system performance, for instance with $R_{t h}^{U}=0.8 \mathrm{Mbps}$ and using the proposed algorithm, the number of served UEs goes from 250 with $d_{0}=100 \mathrm{~m}$ to 100 with $d_{0}=2000 \mathrm{~m}$. This means that starting from a certain distance, a handover to another closer eNB becomes a necessity to maintain the required QoS.

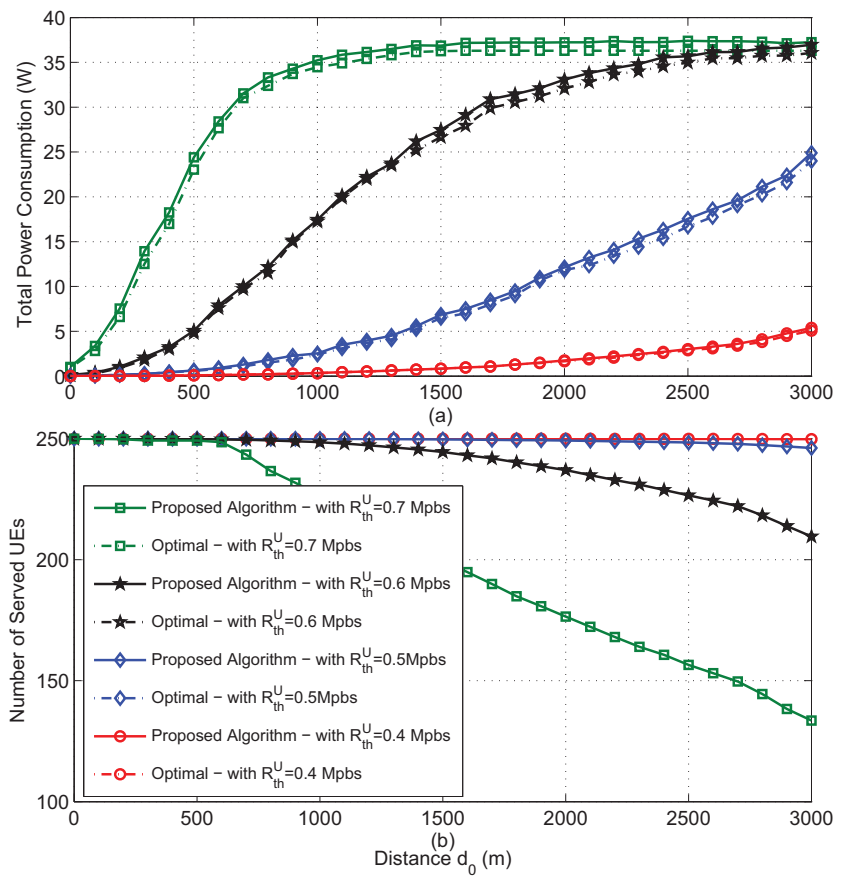

Fig. 5 Performance of the proposed algorithm for the relay transmission versus the distance $d_{0}$ for different values of $R_{t h}^{U}$, (a) Total power consumed by the eNB, (b) Total number of served UEs.

In Fig.5(a), we show that the proposed method (solid lines) consumes a total power almost similar to the derived analytical solution (dashed lines) given in (16). Also, in Fig.5(b), we plot the corresponding number of served UEs as a function of the distance $d_{0}$ for different values of $R_{t h}^{U}=\{0.4,0.5,0.6,0.7\}$ Mbps. We notice that power consumption depends directly on the target data rate and increases with the distance.

\section{CONCLUSION}

This paper studied the problem of resource allocation for high-speed railway with LTE moving relays. Closed form analytical expression of the transmit power allocation was derived. Moreover, we proposed an efficient algorithm based on the Hungarian method to find an optimal resource allocation solution. Interestingly, it has been shown that the performance of the proposed scheme using moving relays can outperform the performance of some previous algorithms as well as the direct transmission. Also, simulation results show that our proposed algorithm maintains the system quality-of-service even for long-range distance with near optimal power consumption.

\section{REFERENCES}

[1] 3rd Generation Partnership Project, "Technical specification group radio access network; Mobile relay for E-UTRA," 3GPP TR 36.836 V 10.0.0, Release 10, Jun. 2012.

[2] Y. Sui, A. Papadogiannis, and T. Svensson, "The potential of moving relays - A performance analysis," in Proc. of the 75th IEEE Vehicular Technology Conference (VTC Spring'2012), Yokohama, Japan, May 2012.

[3] W. Li, C. Zhang, X. Duan, S. Jia, Y. Liu, and L. Zhang, "Performance evaluation and analysis on group mobility of mobile relay for lte advanced system," in Proc. of the 76th IEEE Vehicular Technology Conference (VTC Fall'2012), Quebec, Canada, Sep. 2012.

[4] S. Scott, J. Leinonen, P. Pirinen, J. Vihriälä, V. Phan, and M. Latva-aho, "A cooperative moving relay node system deployment in a high speed train," in Proc. of the 77th IEEE Vehicular Technology Conference (VTC Spring'2013), Dresden, Germany, Jun. 2013.

[5] R. Atat, E. Yaacoub, M. Alouini, and A. Abu-Dayya, "Heterogeneous LTE/802.11a mobile relays for data rate enhancement and energyefficiency in high speed trains," in Proc. of the IEEE Globecom Workshops (GC Workshps'2012), Anaheim, California, USA, Dec. 2012.

[6] 3rd Generation Partnership Project, "Evolved universal terrestrial radio access E-UTRA; Physical channels and modulation,” 3GPP TS 36.211 3GPP V 11.2.0, Release 11, Apr. 2013.

[7] 3rd Generation Partnership Project, "Evolved universal terrestrial radio access E-UTRA; Physical layer procedures," 3GPP TS 36.213 3GPP V 11.2.0, Release 11, Apr. 2013.

[8] P. Kyösti, J. Meinilä, L. Hentilä, X. Zhao, T. Jämsä, C. Schneider, M. Narandzić, M. Milojević, A. Hong, J. Ylitalo, V.-M. Holappa, M. Alatossava, R. Bultitude, Y. de Jong, and T. Rautiainen, "WINNER II Channel Models," Tech. Rep., [Available Online: http://www.istwinner.org.html], Sep. 2007.

[9] Z. Wang, E. Tameh, and A. Nix, "Joint shadowing process in urban peerto-peer radio channels," IEEE Transactions on Vehicular Technology, vol. 57, no.1, pp. 52-64, Jan. 2008.

[10] K. Yamamoto, A. Kusuda, and S. Yoshida, "Impact of shadowing correlation on coverage of multihop cellular systems," in Proc. of the IEEE International Conference on Communications (ICC'2006), Istanbul, Turkey, Jun. 2006.

[11] ETSI TR 101112 (v3.2.0), "Universal mobile telecommunications system (UMTS); Selection procedures for the choice of radio transmission technologies of the UMTS (UMTS 30.03)," Tech. Rep., [Available Online: http://www.etsi.org/deliver/], Apr. 1998.

[12] P.-H. Huang, Y. Gai, B. Krishnamachari, and A. Sridharan, "Subcarrier allocation in multiuser OFDM systems: Complexity and approximability," in Proc. of IEEE Wireless Communications and Networking Conference (WCNC'2010), Sydney, Australia, Apr. 2010.

[13] S. Boyd and L. Vandenberghe, Convex Optimization. New York, NY, USA: Cambridge University Press, 2004.

[14] S. Boyd and A. Mutapcic, "Stochastic Subgradient Methods." Notes for EE364, Stanford University, Winter. 2006-07.

[15] H. W. Kuhn, "The Hungarian Method for the Assignment Problem." 50 Years of Integer Programming 1958-2008. Berlin, Heidelberg. ch. 2, pp. 29-47, 2010.

[16] M. Sternad, M. Grieger, R. Apelfrojd, T. Svensson, D. Aronsson, and A. Martinez, "Using predictor antennas for long-range prediction of fast fading for moving relays," in Proc. of the IEEE Wireless Communications and Networking Conference Workshops (WCNCW'2012), Paris, France, Apr. 2012.

[17] G. Marfia, M. Roccetti, A. Amoroso, and G. Pau, "Safe driving in LA: Report from the greatest intervehicular accident detection test ever," IEEE Transactions on Vehicular Technology, vol. 62, no. 2, pp. 522-535, Feb. 2013. 